\title{
Kit Number
}

National Cancer Institute

\section{Source}

National Cancer Institute. Kit Number. NCI Thesaurus. Code C87822.

A number or sequence of characters that identifies a particular kit. 